\title{
The curved X-ray spectrum of PSR B1509-58 observed with BeppoSAX
}

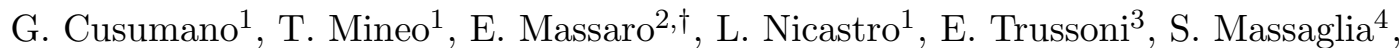 \\ W. Hermsen ${ }^{5}$, and L. Kuiper ${ }^{5}$
}

1 Istituto di Fisica Cosmica ed Applicazioni all'Informatica CNR, Via U. La Malfa 153, 90146 Palermo, Italy

2 Istituto di Astrofisica Spaziale CNR, via Fosso del Cavaliere, 00113 Roma, Italy

3 Osservatorio Astronomico di Torino, Strada Osservatorio 20, 10025 Pino Torinese, Italy

4 Dipartimento di Fisica Generale dell'Università, Via Pietro Giuria 1, 10125 Torino, Italy

5 SRON-Utrecht, Sorbonnelaan 2, 3584 CA Utrecht, The Netherlands

Received 22 October 2000 / Accepted 5 June 2001

\begin{abstract}
We report timing and spectral results for PSR B1509-58 observed by BeppoSAX in February 1998. We obtained pulse profiles with high statistical significance from 0.1 to $300 \mathrm{keV}$ that exhibit the well-known broad asymmetric single pulse. The shape of this pulse does not change across this energy window and can be described as the sum of a narrow and a broader Gaussian component separated $\sim 0.13$ in phase. The spectral distribution can be accurately represented, over the entire BeppoSAX energy range, by a curved function rather than a simple power-law shape. The extrapolation of this model to higher energies is also consistent with the COMPTEL fluxes in the $0.75-30 \mathrm{MeV}$ range, with the maximum luminosity for the broad pulse of PSR B1509-58 reached at $\sim 5.4 \mathrm{MeV}$. The comparison with the Crab pulsar spectrum suggests a possible origin of the X-ray emission in terms of synchrotron radiation from secondary pair particles near the neutron star.
\end{abstract}

Key words. stars: neutron - pulsars: individual: PSR B1509-58 - X-rays: stars

\section{Introduction}

PSR B1509-58 is one of the youngest rotation-powered pulsars, with a period of about $150 \mathrm{~ms}$ and a period derivative of $1.5 \times 10^{-12} \mathrm{~s} \mathrm{~s}^{-1}$, the highest spin-down rate of any known pulsar. Its spin-down age is about 1600 years and the measured braking index is $2.837 \pm 0.001$ (Kaspi et al. 1994). PSR B1509-58 lies in the centre of the supernova remnant MSH 15-52 characterised by a complex structure with thermal and non-thermal components (Tamura et al. 1996). The distance to the pulsar, calculated from the Dispersion Measure (DM), is $5.7 \mathrm{kpc}$ (Taylor \& Cordes 1993), while for MSH 15-52 a distance of $4.2 \mathrm{kpc}$ has been estimated based on neutral hydrogen absorption (Caswell et al. 1975, 1981).

PSR B1509-58 was discovered in the soft X-rays by Einstein (Seward \& Harnden 1982) and soon afterwards detected in the radio band (Manchester et al. 1982). Later on, its pulsed emission was also detected at hard X-ray and soft $\gamma$-ray energies by several satellite and balloonborne experiments: EXOSAT (0.025-11 keV; Trussoni et al. 1990), Ginga (2-60 keV; Kawai et al. 1992), SIGMA

Send offprint requests to: G. Cusumano,

e-mail: cusumano@ifcai.pa.cnr.it

$\dagger$ on leave from Università La Sapienza, Roma, Italy.
(40-300 keV; Laurent et al. 1994), Welcome-1 (94$240 \mathrm{keV}$; Gunji et al. 1994), ROSAT (0.1-2.4 keV; Greiveldinger et al. 1995), ASCA (0.5-10 keV, Saito 1998), BATSE and OSSE aboard the Compton Gamma Ray Observatory (20-5000 keV; Wilson et al. 1993; Matz et al. 1994; Ulmer et al. 1993). Recently, Kuiper et al. (1999) reported definite detection of the pulsed signal in the COMPTEL band up to $10 \mathrm{MeV}$, and of a source compatible with the position of PSR B1509-58 in the skymaps of COMPTEL and EGRET for energies between 10 and $100 \mathrm{MeV}$. A detailed study of the pulse shape and spectrum in the energy range $2-200 \mathrm{keV}$ with the PCA and HEXTE aboard the Rossi X-Ray Timing Explorer has been presented by Marsden et al. (1997) and Rots et al. (1998).

The X-ray pulse profile of PSR B1509-58 is characterised by a broad asymmetric single peak with indications for variations in arrival phase of the pulse maximum with energy (see e.g. Rots et al. 1998; Kuiper et al. 1999). At energies between 10 and $100 \mathrm{MeV}$, Kuiper et al. (1999) reported indications for a double peak structure: a broad peak, aligned with the one observed in X-rays, and a narrower peak at a phase separation of about 0.5 .

The X-ray and soft $\gamma$-ray pulsed spectra have usually been fitted with power laws, but the resulting photon 
indices were generally found to vary with the instrument energy windows. Trussoni et al. (1990) first measured a photon index of about 1.1 with an EXOSAT observation; the same value was found by Saito (1998) with ASCA. Ginga and RXTE observations gave a photon index of 1.35 (Kawai et al. 1993; Marsden et al. 1997), and an even steeper value of 1.68 was derived from BATSE and OSSE observations (Wilson et al. 1992; Matz et al. 1994), suggesting a softening towards the higher energies. However, no evidence for a spectral break was seen in the RXTE data up to $\sim 200 \mathrm{keV}$ (Mardsen et al. 1997). Moreover, a power-law extrapolation of the OSSE results to higher energies is consistent with fluxes measured by COMPTEL up to $\sim 10 \mathrm{MeV}$, but between 10 and $30 \mathrm{MeV}$ the spectrum must break to be consistent with the EGRET results (Kuiper et al. 1999). The latter paper also shows a composite spectrum with most of the earlier measured spectra below $1 \mathrm{MeV}$ discussed above. Large systematic uncertainties are visible, particularly in absolute flux calibration. Such systematic effects could mimic spectral breaks, or could even be due to genuine time variability of the source flux, since the epochs of measurements differ.

In this paper we present the results from simultaneous broad band BeppoSAX observation of PSR B1509-58 and discuss the timing and spectral properties of this pulsar. We will show that the spectral bending is detectable also in the keV range and that the spectral energy distribution, from about $1 \mathrm{keV}$ to the 10-30 MeV COMPTEL energy range, can be well described by a law characterised by a linear dependence of the spectral slope upon the logarithm of energy. The analysis of the extended emission from MSH 15-52 will be presented in another paper (Mineo et al., in preparation).

\section{Observation and data reduction}

PSR B1509-58 was observed on 9-11 February 1998 by the Narrow Field Instruments (NFIs) on board the BeppoSAX satellite (Boella et al. 1997a). The NFIs used in this work were the Low Energy Concentrator Spectrometer (LECS) operating in the energy range 0.1-10 keV (Parmar et al. 1997), the Medium Energy Concentrator Spectrometer (MECS) in the $1.3-10 \mathrm{keV}$ band (Boella et al. 1997b) and the Phoswich Detector System (PDS) between 15 and $300 \mathrm{keV}$ (Frontera et al. 1997). The pulsed flux was also detected by the HPGSPC (Manzo et al. 1997), but these data were not used because of their lower $\mathrm{S} / \mathrm{N}$ ratio.

Standard procedures and selection criteria were applied to the observation data to avoid the South Atlantic Anomaly, solar, bright Earth and particle contamination, using the SAXDAS v.2.0.0 package. The total duration of the observation was $136,265 \mathrm{~s}$ and the net exposure times were $31008 \mathrm{~s}$ for the LECS, $82483 \mathrm{~s}$ for the MECS and 34493 for the PDS. The images in the LECS and MECS show an extended emission region with a roughly elliptical shape, having the major axis at the position angle of $\sim 155^{\circ}$. An intense maximum is evident at the centre of the emission and its position is in full agreement with the radio coordinates of the pulsar, $\alpha=15^{\mathrm{h}} 13^{\mathrm{m}} 55.617$ and $\delta=-59^{\circ} 08^{\prime} 08^{\prime \prime} 87$ (J2000.0) reported by the ATNF Parkes team ${ }^{1}$. In the present work, we are only interested in studying the pulsar emission and to this end, we selected LECS and MECS data within circular regions, centered at the radio position, with radii of $4^{\prime}$ and $3^{\prime}$, respectively. These regions contain $60 \%$ of the LECS and $82 \%$ of the MECS point source distributions. Larger radii would include a higher contribution from the surrounding nebula, reducing the $\mathrm{S} / \mathrm{N}$ ratio.

\section{Timing analysis and pulse profile}

The UTC arrival times of all selected events were first converted to the Solar System Barycentre using the above pulsar position. Then we used the radio ATNF ephemerides to fold the arrival times. An independent check performing a period search through a $\chi^{2}$ maximization gave, for the central time of the observation (MJD 50854.32460), a pulsar period equal to $0.150989037 \mathrm{~s}$, compatible with the radio extrapolation within the period search step size of $5 \times 10^{-9} \mathrm{~s}$.

Phase histograms (120 bins) were obtained for each energy channel of the NFIs. The phase was adjusted to locate the maximum of the pulse at 0.27 , as measured in the RXTE profiles (Rots et al. 1998), to allow the selection of the same phase intervals for the spectral analysis. Pulse profiles in six energy ranges are shown in Fig. 1. The well known single pulse shape is evident in all the energy bands: it is markedly asymmetric, with the leading side much steeper than the trailing one. Notice that the pulse profile in the LECS range is poorly defined because of the relatively low statistics due mainly to the strong absorbing column and the inferior spatial resolution. The marginal feature at phase 0.98, not apparent in the MECS and in the ROSAT profile (Greiveldinger et al. 1995), is likely a statistical fluctuation.

The asymmetric shapes of our pulse profiles, particularly visible with the MECS high statistics, give the suggestion that a "shoulder" is present at phase $\sim 0.4$ on the trailing side of the pulse, consistent with the shape measured by e.g. RXTE (Rots et al. 1998). Kuiper et al. (1999) noted that the profile measured by COMPTEL (0.75-10 MeV) appeared to be shifted in phase with respect to the RXTE profile, with its maximum around the phase of the shoulder. In an attempt to explain this apparent variation in pulse shape with energy, they investigated whether the asymmetric shapes of the pulse profiles can be interpreted as being produced by the superposition of two components having a rather narrow phase separation. Indeed, the RXTE (2-16 keV) profile can be well represented by the sum of two Gaussians, a narrow component peaking at phase $0.250 \pm 0.008$ and with width $0.056 \pm 0.008$ and a broader one at $0.386 \pm 0.012$ and

\footnotetext{
1 available from http://www.atnf .csiro.au/research/ pulsar/psr/archive/
} 


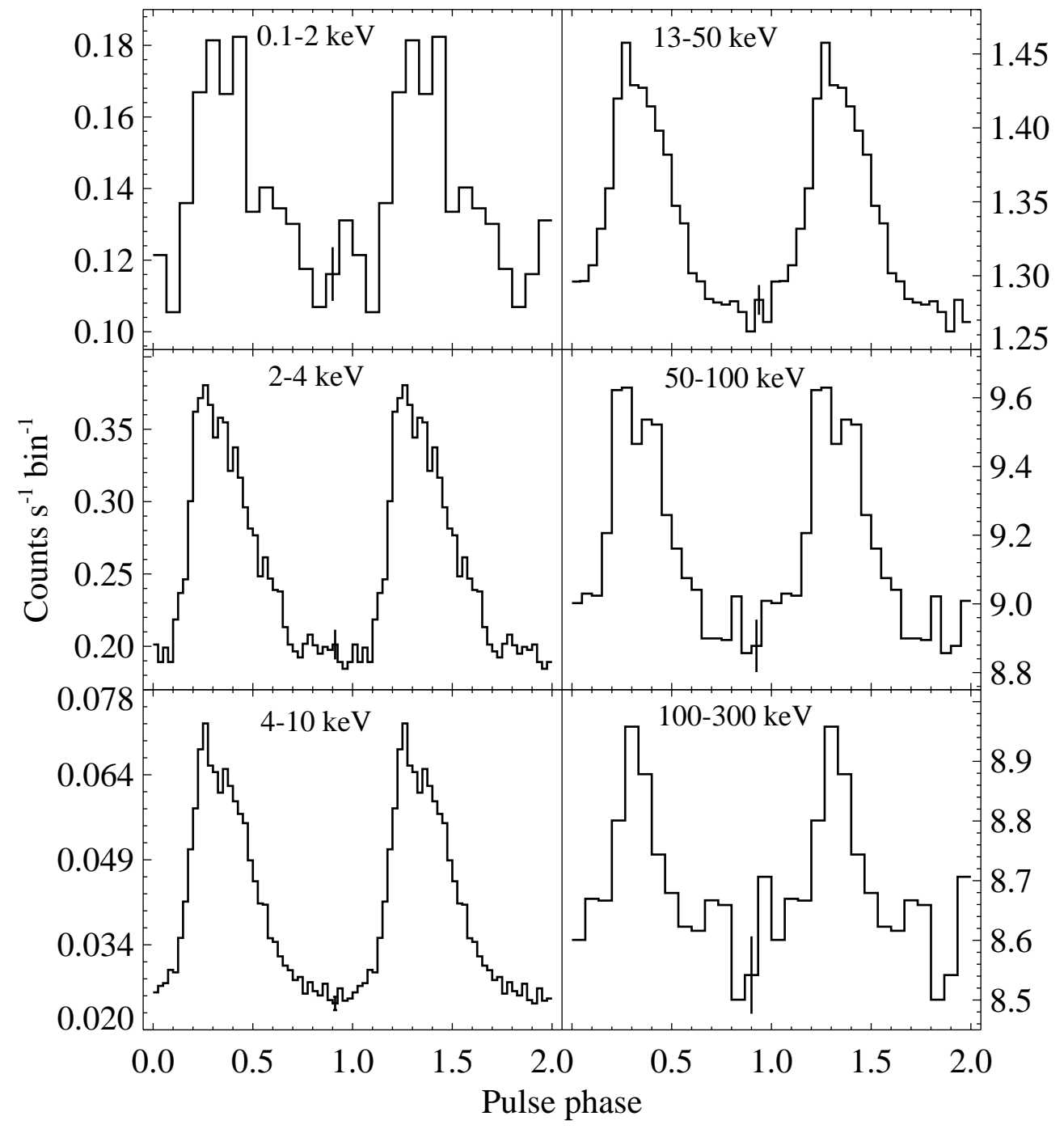

Fig. 1. Pulse profiles of PSR B1509-58 in six energy bands from the LECS(0.1-2 keV), MECS(1.6-4 keV and 4-10 keV) and PDS (13-50 keV, 50-100 keV and 100-300 keV). Two phase intervals are reported for clarity. Typical $1 \sigma$ error bars are shown.

having a width of $0.129 \pm 0.006$. The narrow pulse accounted for $25.7 \pm 4.3 \%$ of the RXTE total pulsed emission. Applying a similar fit to the COMPTEL profile (0.75-10 MeV), but fixing positions and widths to the values obtained for the RXTE profile, Kuiper et al. (1999) found that the first narrow pulse accounts for $13 \pm 18 \%$ of the total pulsed emission, consistent therefore with being absent, given the profile satisfactorily described by just the broad second pulse near 0.39 .

We checked the consistency of our MECS and PDS profiles with the scenario of two pulse components with relative strengths varying with energy. Therefore, we applied the same model to the MECS and PDS profiles, integrated over their respective entire energy ranges to improve the statistics. We found good reduced $\chi^{2}$ values, quite close to unity. The resulting parameters for the MECS were: the central phases of the two Gaussians $0.243 \pm 0.003$ and $0.382 \pm 0.006$, their sigmas $0.051 \pm 0.004$ and $0.137 \pm 0.004$, in agreement to better than one standard deviation with the results of Kuiper et al. (1999). For the PDS central phases we obtained $0.254 \pm 0.006$ and $0.351 \pm 0.007$, while their sigmas were $0.033 \pm 0.006$ and $0.144 \pm 0.007$. The first components account for $20 \pm 3 \%$ and $9 \pm 2 \%$ of the total pulsed emission for MECS and PDS data, respectively, suggesting that the transition from the RXTE pulse shape to the COMPTEL one already takes place over the MECS-PDS energy range. However, the phase separation between the two Gaussian components changes from 0.14 for the MECS to about 0.10 for the PDS. In addition, the width of the first Gaussian component is much narrower in the PDS profile than in the MECS one. We stress that these differences depend strongly upon the centroid positions. Fixing the central phases to the values reported by Kuiper et al. (1999), we found an acceptable $\chi^{2}$ value, within 2 standard deviations, and parameter values quite similar for both energy windows: the widths of the two Gaussians become $0.055 \pm 0.003$ and $0.139 \pm 0.004$ for the MECS and $0.057 \pm 0.009$ and $0.128 \pm 0.009$ for the PDS. The resulting model profiles are shown in Fig. 2. In this case the first component account for $22.3 \pm 1.4 \%$ and $24 \pm 4 \%$ of the total pulsed emission for MECS and PDS data, 
respectively, fully in agreement with the RXTE values $(2-16 \mathrm{keV})$ found by Kuiper et al. (1999). This analysis suggests that the pulse shapes and flux ratios of the two possible components do not change between these two energy bands. Therefore, the transition from the hardX-ray pulse profile shape (PDS below $300 \mathrm{keV}$ ) to the COMPTEL 1-10 MeV $\gamma$-ray profile should occur above the PDS energy window.

\section{Spectral analysis}

The evaluation of the pulsed spectrum is not a simple statistical task because of the presence of a high unpulsed level. Two different methods can be followed. The first is that of subtracting the mean off-pulse counts in each energy bin from the pulsed signal and adding together some bins in order to have a significant number of events; the second way is to include in the fit of the pulsed counts the spectral law derived from the off-pulse best fit. In the former case, the number of degrees of freedom is smaller and the $\chi^{2}$ can be biased towards lower values, because the variance is increased by a factor of about two due to the off-pulse subtraction. In the latter case, indeterminations in the dc model cannot simply be taken into account. In our analysis we followed both these methods and obtained the same results.

In the spectral analysis, the pulsed and the off-pulse spectra were extracted from the phase interval $0.17-0.53$ and $0.77-1.07$, respectively, adopting the phase definitions of Marsden et al. (1997). Normalization factors, taking into account the intercalibration systematics, have been included in the model when simultaneously fitting spectra from different BeppoSAX instruments. The quoted errors represent the statistical $1 \sigma$ confidence level.

\subsection{The $N_{\mathrm{H}}$ estimate}

A good knowledge of the $N_{\mathrm{H}}$ value in the direction of PSR B1509-58 is important for the spectral analysis. Several literature estimates (Greiveldinger et al. 1995; Trussoni et al. 1996; Saito 1998) are consistent with a value around $9 \times 10^{21} \mathrm{~cm}^{-2}$, while higher estimates were derived from RXTE observations: $1.27 \times 10^{22} \mathrm{~cm}^{-2}$ (Marsden et al. 1997) and the much higher value of $6.0 \times 10^{22} \mathrm{~cm}^{-2}$ given by Rots et al. (1998).

To evaluate the column density, we analysed the LECS and MECS spectra of the off-pulse region assuming that it mainly originates from the nebula at the same distance as the pulsar. The use of the nebular spectrum has the advantage of good statistics. The fit with an absorbed power law to the LECS and MECS data in the energy range $0.1-10.0 \mathrm{keV}$ gives a spectral index of $1.89 \pm 0.03$ and $N_{\mathrm{H}}$ of $(9.1 \pm 0.5) \times 10^{21} \mathrm{~cm}^{-2}$ with a reduced $\chi^{2}$ of 1.0 (d.o.f. 160). This column density agrees well with the ROSAT and ASCA results and, furthermore, it is only $15 \%$ higher than the estimate derived from the DM in the
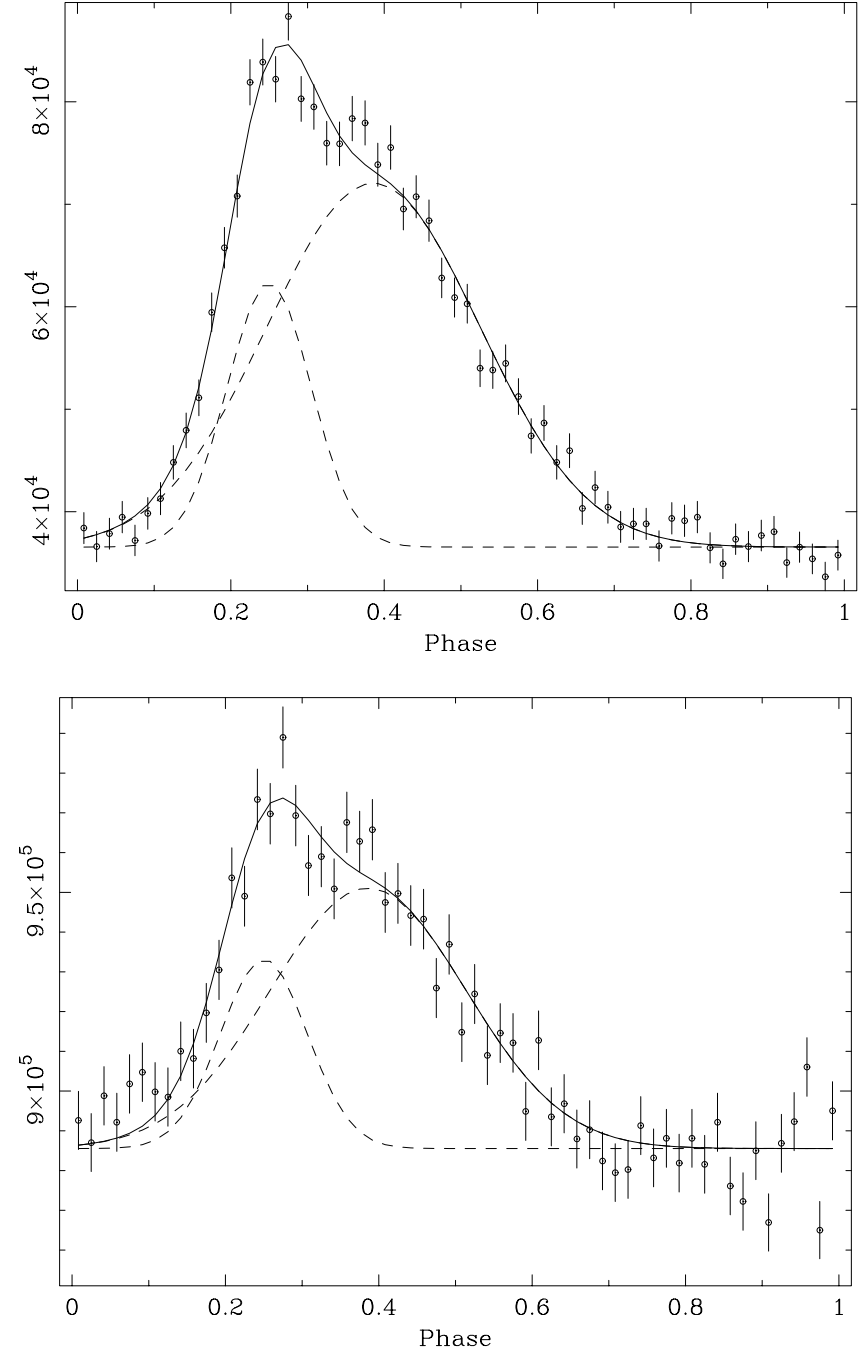

Fig. 2. The two-Gaussian model components and their sum compared with the observed pulse profiles of PSR B1509-58 in the MECS (1.6-10 keV; upper) and PDS (15-300 keV; lower) ranges. The best fit was obtained by fixing the central phases of the two Gaussians to the values reported by Kuiper et al. (1999).

pulsar direction (253.2 pc cm ${ }^{-3}$; Kaspi et al. 1994) assuming 10 neutral hydrogen atoms per free electron (Clark \& Caswell 1976). The only $N_{\mathrm{H}}$ estimate largely different from ours is that derived by Rots et al. (1998) from a spectral fit near the peak of the pulse using RXTE data at energies greater than $2 \mathrm{keV}$. We think that the latter value is too high and this discrepancy might be explained either by the higher energy range used in their analysis, hardly constraining $N_{\mathrm{H}}$, and/or by an intrinsic curvature of the pulsed spectrum, as we will show in the next section. We also extended the power law fit including the PDS data up to $300 \mathrm{keV}$ and found again the photon index of $1.89 \pm 0.02$ with a reduced $\chi^{2}$ of 0.99 (d.o.f. 173), confirming that the same slope well represents the nebular spectrum, also at such higher energies. 


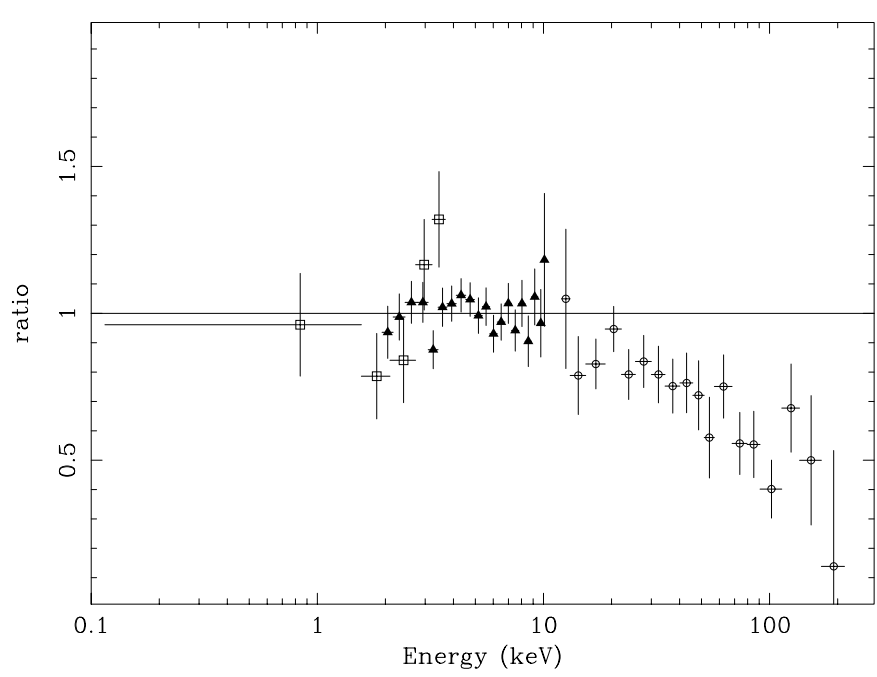

Fig. 3. LECS (square), MECS (triangle) and PDS (circle) data divided by the folded model extrapolated, over the entire BeppoSAX range, from the MECS spectrum of Table 1.

\subsection{The pulsed spectrum}

First, we fitted simultaneously the pulsed spectra of the NFIs with a single power law after subtraction of the off-pulse level and binning the energy channels to have a minimum of 20 counts in each individual energy bin. The relative intercalibration factors of the LECS and PDS with respect to the MECS were left free, whereas the column density was fixed at the $N_{\mathrm{H}}$ value derived above for the off-pulse phase interval. This fit did not give a satisfactory result for the spectral distribution over the entire BeppoSAX range. The resulting reduced $\chi^{2}$ was 1.28 for 37 d.o.f., but the intercalibration factors were found outside the expected ranges. More precisely, the resulting PDS-MECS intercalibration factor was 0.74 , while the expected values range between 0.83 and 0.89 (Fiore et al. 1999) and that between LECS and MECS was 0.61, while values between 0.7 and 1.0 are expected. On the other hand, fixing these parameters to values within the expected ranges, the $\chi_{\nu}^{2}$ becomes worse, being always greater than 1.45 (39 d.o.f.).

Better fits are obtained when the NFIs are considered separately. In Table 1 we report for each instrument the energy range, the resulting photon index with the statistical uncertainty and the reduced $\chi^{2}$. Notice that the photon index for the PDS is significantly larger than that for the MECS, while that derived for the LECS is smaller (flatter spectrum). In Fig. 3 we reported the NFIs data divided by the folded model extrapolated, over the entire BeppoSAX range, from the MECS spectrum (that with the highest $\mathrm{S} / \mathrm{N}$ ratio) using the best fit values given in Table 1 . The PDS data are systematically below the MECS extrapolation, reflecting the different spectral shape in the two instruments' energy range. A single power law shape is obviously not an appropriate representation of the pulsar spectrum over such a wide energy range.
Table 1. Power-law fit parameters for each single NFI.

\begin{tabular}{cccc}
\hline Instrument & $\begin{array}{c}\text { Range } \\
(\mathrm{keV})\end{array}$ & Photon index & $\chi_{\nu}^{2}$ (d.o.f.) \\
\hline LECS & $0.1-4$ & $0.7 \pm 0.2$ & $0.88(3)$ \\
MECS & $1.6-10$ & $1.19 \pm 0.04$ & $0.67(18)$ \\
PDS & $15-300$ & $1.48 \pm 0.06$ & $0.70(14)$ \\
\hline
\end{tabular}

The spectral fits obtained without the off-pulse subtraction but considering two power laws at the same time, one of which has the spectral parameters of the off-pulse frozen at the values given in Sect. 4.1 , did not give a better result. The resulting reduced $\chi^{2}$ was 1.27 but now with 261 d.o.f., and therefore higher that the expected value by more than three standard deviations.

Analogous to the Crab Pulsar (Massaro et al. 2000), we interpreted all these results as an indication that a mildly curved spectral distribution gives a better description of the combined spectrum than a single-power-law shape. Therefore, we fitted the combined BeppoSAX spectrum of PSR B1509-58 with a law characterised by a linear dependence of the spectral slope upon the logarithm of energy:

$F(E)=K\left(E / E_{0}\right)^{-\left[a+b \log \left(E / E_{0}\right)\right]}$.

Of course, several other laws can be considered, but this has the advantage of a simple analytical expression with only one parameter more than a single power law. In our fit $E_{0}$ was fixed at $1 \mathrm{keV}$. The energy-dependent photon in$\operatorname{dex} \alpha(E)$ can be evaluated by the log derivative of Eq. (1):

$\alpha(E)=a+2 b \log \left(E / E_{0}\right)$.

The best fit of Eq. (1) to the subtracted counts, fixing the $N_{\mathrm{H}}$ value to the results derived in Sect. 4.1, gave a much better reduced $\chi^{2}$ equal to 0.74 (36 d.o.f.). The F-test probability for such an improvement by chance with respect to the first result is $0.9 \times 10^{-5}$. Furthermore, the resulting instrumental intercalibration factors $0.87 \pm$ 0.07 for PDS-MECS and $0.67 \pm 0.05$ for LECS-MECS are now consistent with the expected values. The values obtained for the three spectral parameters are: $a=$ $0.96 \pm 0.08, b=0.16 \pm 0.04$ and $K=(1.8 \pm 0.3) \times$ $10^{-3} \mathrm{ph} \mathrm{cm}^{-2} \mathrm{~s}^{-1} \mathrm{keV}^{-1}$. The fitted spectrum and the residuals are shown in Fig. 4. Note that the systematic trend, in particular for the LECS and PDS, are not present anymore. The pulsed flux in the $2-10 \mathrm{keV}$ range is $2.0 \times 10^{-11} \mathrm{erg} \mathrm{cm}^{-2} \mathrm{~s}^{-1}$ and that in the $2-300 \mathrm{keV}$ range is $2.3 \times 10^{-10} \mathrm{erg} \mathrm{cm}^{-2} \mathrm{~s}^{-1}$, corresponding to an isotropic luminosity of about $7 \times 10^{35} \mathrm{erg} \mathrm{s}^{-1}$ for an assumed distance of $5 \mathrm{kpc}$.

The fit of the same law added to the off-pulse power spectrum reduced the $\chi^{2}$ again to 0.94 , but such a decrease has a much larger statistical F-test significance (the chance probability is now less than $10^{-18}$ ) because the d.o.f. number is 261 . The best fit values of $a$ and $b$ were $0.90 \pm 0.05$ and $0.21 \pm 0.03$, respectively, in agreement with the previous results within a standard deviation. 


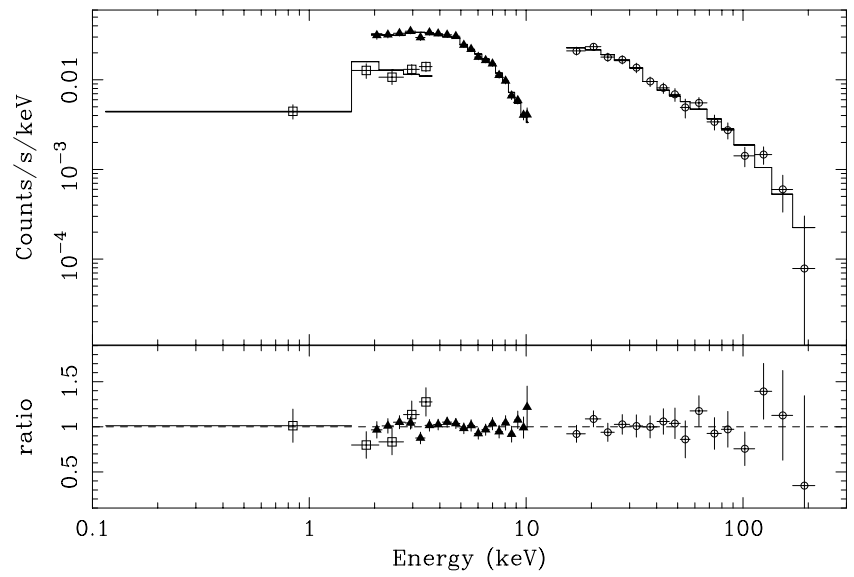

Fig. 4. The energy spectrum of PSR B1509-58 obtained from the LECS, MECS and PDS, fitted with the model expressed by Eq. (1).

Extrapolation of the simple power-law model to higher energies predicts fluxes significantly higher than the values measured by OSSE, BATSE and COMPTEL (Wilson et al. 1993; Matz et al. 1993; Ulmer et al. 1993; Kuiper et al. 1999), while the spectral distribution of Eq. (1) agrees much better with the higher-energy data. We verified this agreement by fitting Eq. (1) to the combined deconvolved BeppoSAX spectra together with the COMPTEL 0.75-30 MeV spectrum for the same broad pulse (Fig. 9 in Kuiper et al. 1999). The values of the fit parameters remain the same within their uncertainties $(a=1.03 \pm 0.05, b=0.13 \pm 0.02$ and $K=(1.9 \pm$ $0.1) \times 10^{-3} \mathrm{ph} \mathrm{cm}^{-2} \mathrm{~s}^{-1} \mathrm{keV}^{-1}$ ), and Fig. 5, where the Spectral Energy Distribution (SED) of the pulsed is plotted, shows that we get a good fit up to $30 \mathrm{MeV}$. Flux upper limits derived from timing analysis of EGRET data (Thompson, private communication) are also shown in the figure. These upper limits do not constrain our fit with Eq. (1) significantly, but the indications for detections above $100 \mathrm{MeV}$ in spatial analysis of EGRET data by Kuiper et al. (1999) give fluxes below the extrapolation of Eq. (1).

It is interesting to note that the SED of the pulsed emission, according to this spectral law, should have a maximum at the energy:

$E_{\mathrm{m}}=E_{0} 10^{[(2-a) /(2 b)]}$,

which, with the values for the best fit to the combined BeppoSAX and COMPTEL data, corresponds to $\sim 5.4 \mathrm{MeV}$ in agreement with the maximum of the COMPTEL spectrum shown in Fig. 5.

We can thus conclude that the wide band SED of PSR B1509-58 is actually well represented by a curved spectrum over an energy interval about four orders of magnitude wide $(\sim 1 \mathrm{keV}$ up to $\sim 30 \mathrm{MeV}$; Fig. 5$)$.

\section{Discussion}

The wide band BeppoSAX observation of PSR B1509-58, with simultaneous measurements of energies from less

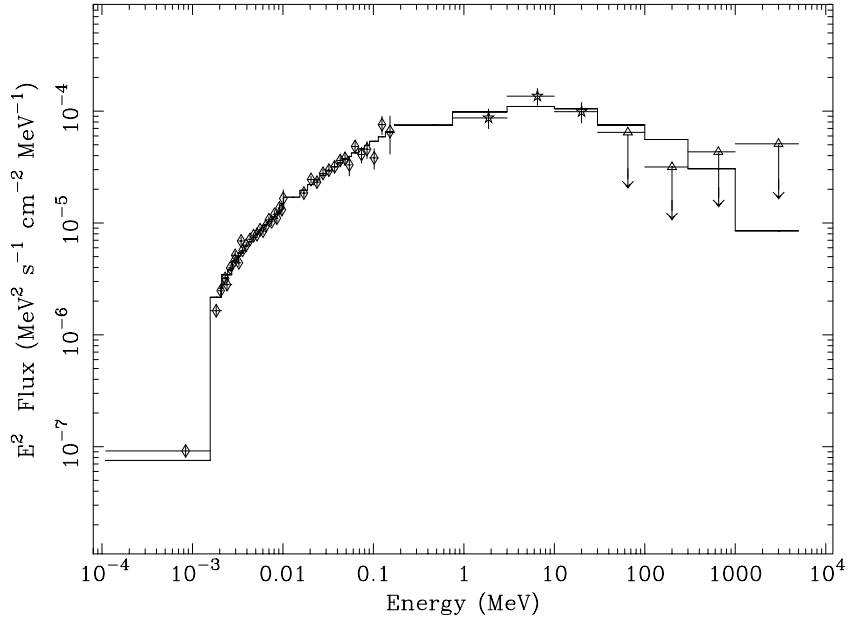

Fig. 5. The deconvolved SED of the pulsed emission from PSR B1509-58. Data are from BeppoSAX (diamond), COMPTEL (star) and EGRET upper limits (triangle). The solid line represents the best fit with Eq. (1) to the BeppoSAX and COMPTEL data.

than $1 \mathrm{keV}$ up to $\sim 300 \mathrm{keV}$, allows a detailed study of the pulsar spectral energy distribution and of its broad asymmetric pulse profile.

For the energy windows of the MECS $(1.6-10 \mathrm{keV})$ and the PDS $(15-300 \mathrm{keV})$ we derived phase distributions which appeared to be identical in shape, within the statistical uncertainties. Both pulse profiles can be accurately fitted with two Gaussian components, a narrow one centered at phase $\sim 0.25$ and width $\sim 0.056$, and a broader component at phase $\sim 0.38$ and width $\sim 0.13$. These values are fully consistent with those reported by Kuiper et al. (1999) for the RXTE pulse profile between 2 and $16 \mathrm{keV}$. In addition, for all three profiles, the first narrow component contributes $\sim 22 \%$ to the total pulsed flux, justifying the conclusion that the pulse profile is, to a high degree of accuracy, constant in shape over the range $\sim 1-300 \mathrm{keV}$. Kuiper et al. (1999) showed that, for energies between 0.75 and $10 \mathrm{MeV}$, the profile can also be explained with the same two Gaussian components, but the first component seems to be suppressed, shifting the maximum of the total pulse to a higher pulse phase. Apparently, the change in pulse shape occurs above the PDS energy range.

In the spectral analysis, we first derived an accurate estimate of the column density, using only the off-pulse spectrum. Our result of $9.1 \times 10^{21} \mathrm{~cm}^{-2}$ is in agreement with several previous estimates but not with the high value derived by Rots et al. (1998) from RXTE data. We showed that a good representation of the overall spectral distribution of the pulsed emission can be achieved with a second order law in the double log plot, like Eq. (1). The conclusion that the spectrum of PSR B1509-58 is most likely curved was also drawn in previous studies. Matz et al. (1994), for instance, suggested this possibility but their data did not allow them to decide whether the changes in the power-law index occur at discrete energies, like a break, or over a broad energy range. Futhermore, 
systematic differences in the absolute sensitivities among instruments operating in different energy windows create discontinuities when combining the spectra, which were generally collected at different epochs. The BeppoSAX NFIs view the pulsar simultaneously, thus intrinsic time variability cannot hamper the interpretation, although we should note that the high-energy emission from radio pulsars appeared so far to be stable.

We have shown that the curved model gives a satisfactory description of the PSR B1509-58 spectrum from BeppoSAX data up to the COMPTEL energy window 0.75-30 MeV. Above $30 \mathrm{MeV}$, the genuine spectrum of PSR B1509-58 has to break more abruptly than described by Eq. (1), as seems to be required by the EGRET results above $100 \mathrm{MeV}$ (see Kuiper et al. 1999).

PSR B1509-58 and the Crab are the two pulsars for which the spectra are best determined between $1 \mathrm{keV}$ and $30 \mathrm{MeV}$. The only other pulsar observed in this energy window is the Vela pulsar (Strickman et al. 1999), but its measured spectrum has lower statistics. Therefore, it seems interesting to make a comparison with the Crab pulsar. A recent pulse shape and spectral analysis of BeppoSAX Crab data (Massaro et al. 2000, 2001) has shown that the X-ray emission can be explained by the superposition of two components with different spectral and phase distribution. The SED of these components can be well fitted by a function like Eq. (1). The values of the parameter $b$ for these two components were practically equal to that of PSR B1509-58, while those of $a$ resulted equal to 1.63 and 1.31 for the first peak and the interpeak components, respectively. Such curved spectra suggest the existence of characteristic energies, for instance that of the SED peak. In the previous section we showed that for PSR B1509-58, fitting BeppoSAX and COMPTEL data simultaneously, it is equal to $\sim 5 \mathrm{MeV}$. For the two components of Crab the first peak SED has a maximum at $\sim 14 \mathrm{keV}$, while that of the interpeak lies at $\sim 200 \mathrm{keV}$.

One can reasonably ask whether the energy of the SED maximum has, at least for Crab-like pulsars, some simple scaling law with the two most important observable quantities: the period and its first derivative. In the framework of the outer gap model, originally developed by Cheng et al. (1986a, 1986b), curvature gamma-rays are converted into electron-positron pairs by the interaction with the magnetic field. X-ray photons are then radiated by these secondary particles as synchrotron emission.

The typical energy of primary curvature photons can be estimated by

$E_{\text {cur }} \simeq 10^{8} f^{3 / 2} P^{-7 / 4} B_{* 12}^{3 / 4} \quad \mathrm{eV}$,

where $f$ is the fractional volume of the outer magnetosphere occupied by the outer gap and $B_{* 12}$ is the magnetic field at the neutron star surface in units of $10^{12}$ Gauss (Cheng \& Wei 1995). Zhu \& Ruderman (1997) and Wang et al. (1998) proposed that pairs are copiously produced in the near surrounding of the neutron star by curvature photons radiated by inward moving electrons. Assuming that the cut-off energy of the pair particles is about $E_{\text {cur }} / 2$, the energy of synchrotron photons would result in

$E_{s} \simeq\left(e \hbar / 4 m^{3} c^{5}\right) E_{\mathrm{cur}}^{2} B$

A well established formula for $f$ in terms of the main pulsar parameters is not yet available and different relations can be found in the literature. Using the scaling formula given by Cheng et al. (1986a - Eq. (6.7)) for Crab-like pulsars

$f \simeq 0.1\left(B_{*} / B_{* \mathrm{C}}\right)^{-13 / 20}\left(P / P_{\mathrm{C}}\right)^{33 / 20}$,

where the index $\mathrm{C}$ is referred to the Crab values, and considering that near the neutron star the magnetic field can be considered proportional to $(P \dot{P})^{1 / 2}$, we can derive the following approximate scaling relation for the energy of the maximum in the synchrotron spectrum with respect to Crab:

$$
\begin{aligned}
\left(E_{\mathrm{m}} / E_{\mathrm{mC}}\right) & \simeq\left(P / P_{\mathrm{C}}\right)^{69 / 40}\left(\dot{P} / \dot{P}_{\mathrm{C}}\right)^{11 / 40} \\
& \simeq\left(P / P_{\mathrm{C}}\right)^{7 / 4}\left(\dot{P} / \dot{P}_{\mathrm{C}}\right)^{1 / 4}
\end{aligned}
$$

With the well-known timing parameter values of PSR B1509-58 and Crab, this ratio turns out to be $\sim 20$. It agrees quite well when the spectrum of the Crab interpeak region is considered, while it is about one order of magnitude greater for that of the first peak of Crab. These results, although only indicative, suggest that the X-ray emission of PSR B1509-58 and that of the Crab interpeak component can have the same origin, likely being synchrotron radiation from secondary pairs. Another interesting similarity between these emission components of the two pulsars and supporting this interpretation is that both are not detected in the EGRET range.

Recently Zhang \& Cheng (2001) extended the work by Cheng et al. (2000) and computed some pulse shapes and spectra of PSR B1509-58 in the X- and $\gamma$-ray ranges on the basis of a synchrotron self-Compton mechanism in a finite region just above the outer gaps. The resulting spectral cut-offs lie at energies less than about $1 \mathrm{MeV}$, smaller than that found by the present analysis. According to their assumptions, however, from the soft X-ray band to a few hundred $\mathrm{keV}$ the spectrum is a single power law with a photon index of 1.5 , similar to that found by us in the PDS range but much steeper than that of the MECS. Furthermore, their pulse shape is characterised by a single broad peak but with a duty cycle about equal to 0.25 , while the measured one is about twice as wide (see Fig. 2). In these papers, a different formula for the volume fraction occupied by the outer gap is also used and a different scaling relation for the synchrotron photon energies is obtained. The ratio with the second peak of Crab lowers to about 4, which would imply a cutoff energy for PSR B1509-58 around $1 \mathrm{MeV}$. More accurate observational data and more detailed models are required to resolve this issue.

The hard X/soft $\gamma$-ray spectrum of PSR B1509-58 has also been discussed in detail in the context of polar 
cap models, in particular considering the exotic photonsplitting attenuation process (Harding et al. 1997) in the very strong polar surface magnetic field strength of $\sim 3.1 \times 10^{13}$ Gauss. In Kuiper et al. (1999) it was shown that the combined COMPTEL/EGRET spectral results are consistent with such an interpretation for a magnetic co-latitude of the emission rim of $\sim 2^{\circ}$, close to the classical radius of the polar cap. Our curved BeppoSAX spectrum is approximately consistent with the model calculations of Harding et al. (1997), who considered for simplicity only cases for a single co-latitude, i.e. a polar rim rather than an extended cap. More refined model calculations should indicate whether the above model predicts the smooth curvature found in our analysis.

As mentioned above, for only three pulsars, the spectra were measured over the broad BeppoSAX energy window up to the COMPTEL MeV $\gamma$-rays. The present analysis confirms that such wide band spectral observations are very important for the study of the SED of radio pulsars, and give us the possibility to search e.g. for characteristic energies. Future space missions sensitive over this hard X-ray/soft $\gamma$-ray band, particularly ESA's INTEGRAL mission which is due for launch in 2002, should include the study of these sources among their primary scientific targets.

Acknowledgements. We are grateful to M. Ruderman and M. Salvati for their useful comments. The CNR Institutes and the BeppoSAX Science Data Center are financially supported by the Italian Space Agency (ASI) in the framework of the BeppoSAX mission. E. T., S. M. and E. M. acknowledge the Italian Ministry of University and Scientific Research (grant Cofin 99-02-02) the Italian Space Agency (ASI) and the MURST for financial support.

\section{References}

Boella, G., Butler, R. C., Perola, G. C., et al. 1997a, A\&AS, 122,299

Boella, G., Chiappetti, L., Conti, G., et al. 1997b, A\&AS, 122, 327

Caswell, J. L., Murray, J. D., Roger, R. S., Cole, D. J., \& Cooke, D. J. 1975, A\&A, 45, 239

Caswell, J. L., Milne, D. K., \& Wellington, K. J. 1981, MNRAS, 195,557

Cheng, K. S., Ho, C., \& Ruderman, M. 1986a, ApJ, 300, 500

Cheng, K. S., Ho, C., \& Ruderman, M. 1986b, ApJ, 300, 522

Cheng, K. S., Ruderman, M., \& Zhang, L. 2000, ApJ, 537, 964

Clark, D. H., \& Caswell, J. L. 1976, MNRAS, 174, 267

Fiore, F., Guainazzi, M., \& Grandi, P. 1999, Cookbook for BeppoSAX NFI Spectral Analysis, http://www.sdc.asi.it/software
Frontera, F., Costa, E., Dal Fiume, D., et al. 1997, A\&AS, 122, 357

Greiveldinger, G., Caucino, S., Massaglia, S., Ogelman, H., \& Trussoni, E. 1995, ApJ, 454, 855

Gunji, S., Hirayama, M., Kamae, T., et al. 1994, ApJ, 428, 284

Harding, A., Baring, M., \& Gonthier, P. 1997, ApJ, 476, 246

Hartmann, D. H., Brown, L. E., Clayton, D. D., et al. 1993, in Isolated Pulsar, ed. K.A. Van Riper, R. Epstein, \& C. Ho (Cambridge Uni. Press), 405

Kaspi, V. M., Manchester, R. N., Siegman, B., Johnston, S., \& Lyne, A. G. 1994, ApJ, 422, L83

Kawai, N., Okayasu, R., \& Sekimoto, Y. 1992, in AIP Conf. Proc. 280, Proc. Workshop Compton Observatory, ed. M. Friedlander, N. Gehrels, \& D. J. Macomb (New York)

Kuiper, L., Hermsen, W., Krijger, J. M., et al., 1999, A\&A, 351, 119

Laurent, P., Paul, J., Claret, A., et al. 1994, A\&A, 286, 838

Manchester, R. W., Tuohy, I. R., \& D'Amico, N. 1982, ApJ, 262, L31

Manzo, G., Giarrusso, S., Santangelo, A., et al. 1997, A\&AS, 122,341

Marsden, D., Blanco, P. R., Gruber, D. E., et al. 1997, ApJ, 491, L39

Massaro, E., Litterio, M., Cusumano, G., \& Mineo, T. 2000, A\&A, 361, 695

Massaro, E., Litterio, M., Cusumano, G., \& Mineo, T. 2001, 4th INTEGRAL Workshop, Alicante, Spain, in press

Matz, S. M., Ulmer, M. P., Grabelsky, D. A., et al. 1994, ApJ, 434, 288

Parmar, A. N., Martin, D. D. E., Bavdaz, M., et al. 1997, A\&AS, 122, 309

Rots, A. H., Jahoda, K., Macomb, D. J., et al. 1998, ApJ, 501, 749

Saito, Y. 1998, Ph.D. Thesis

Seward, F. D., \& Harnden, F. R. 1982, ApJ, 256, L45

Strickman, M. S., Harding, A. K., \& de Jager, O. C. 1999, ApJ, 524,373

Ulmer, M. P., Matz, S. M., Wilson, R. B., et al. 1993, ApJ, 417, 738

Tamura, K., Kawai, N., Yoshida, A., \& Brinkman, W. 1996, PASJ, 48, L33

Taylor, J. H., \& Cordes, J. M. 1993, ApJ, 411, 674

Trussoni, E., Brinkmann, W., Ögelman, H., et al. 1990, A\&A, 234,403

Trussoni, E., Massaglia, S., Caucino, S., Brinkmann, W., \& Aschenbach, B. 1996, A\&A, 306, 329

Wang, F. Y.-H., Ruderman, M., Halpern, J. P., \& Zhu, T. 1998, ApJ, 498, 373

Wilson, R. B., Finger, M. H., Pendlenton, G. N., et al. 1993, in Isolated Pulsar, ed. K. A. Van Riper, R. Epstein, \& C. Ho (Cambridge Univ. Press), 257

Zhang, L., \& Cheng, K. S. 2001, A\&A, 363, 575

Zhu, T., \& Ruderman, M. 1997, ApJ, 478, 701 